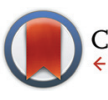

CrossMark

Cite this: Food Funct., 2016, 7, 3989

\title{
Comparison of lipases for in vitro models of gastric digestion: lipolysis using two infant formulas as model substrates
}

\author{
P. J. Sassene, ${ }^{a}$ M. Fanø, ${ }^{b}$ H. Mu, ${ }^{a}$ T. Rades, ${ }^{a}$ S. Aquistapace, ${ }^{c}$ B. Schmitt, ${ }^{c}$ \\ C. Cruz-Hernandez, ${ }^{c}$ T. J. Wooster ${ }^{c}$ and A. Müllertz ${ }^{\star a, b}$
}

\begin{abstract}
The aim of this study was to find a lipase suitable as a surrogate for Human Gastric Lipase (HGL), since the development of predictive gastrointestinal lipolysis models are hampered by the lack of a lipase with similar digestive properties as HGL. Three potential surrogates for HGL; Rhizopus Oryzae Lipase (ROL), Rabbit Gastric Lipase (RGL) and recombinant HGL ( $r H G L)$, were used to catalyze the in vitro digestion of two infant formulas (a medium-chain triacylglyceride enriched formula (MC-IF) and a predominantly long-chain triacylglyceride formula (LC-IF)). Digesta were withdrawn after 0, 5, 15, 30, 60 min of gastric digestion and after 90 or 180 min of intestinal digestion with or without the presence of pancreatic enzymes, respectively. The digesta were analyzed by scanning electron microscopy and gas chromatography to quantify the release of fatty acids (FAs). Digestions of both formulas, catalyzed by ROL, showed that the extent of gastric digestion was higher than expected from previously published in vivo data. ROL was furthermore insensitive to FA chain length and all FAs were released at the same pace. RGL and rHGL favoured the release of MC-FAs in both formulas, but $r H G L$ did also release some LC-FAs during digestion of MC-IF, whereas RGL only released MC-FAs. Digestion of a MC-IF by HGL in vivo showed that MC-FAs are preferentially released, but some LC-FAs are also released. Thus of the tested lipase rHGL replicated the digestive properties of $\mathrm{HGL}$ the best and is a suitable surrogate for HGL for use in in vitro gastrointestinal lipolysis models.
\end{abstract}

Received 5th February 2016 Accepted 11th August 2016 DOI: $10.1039 / \mathrm{c} 6 f \circ 00158 \mathrm{k}$ www.rsc.org/foodfunction pre-digestion by gastric lipase which helps to distrupt their native milk fat globule membrane. ${ }^{12,13}$ Given the important role that gastric lipase plays in infants and adults with pancreatic insufficiency it is important to have in vitro models that accurately replicate its action.

Several different digestion models have been developed to simulate lipid digestion occurring throughout the gastrointestinal (GI)-tract in order to evaluate nutritional and pharmaceutical products. The majority of these in vitro lipolysis models are only taking the intestinal digestion into account. ${ }^{16-28}$ This is due to several reasons of which the most crucial one is that the majority of lipid digestion occurs in the proximal part of the small intestine. The throughput of the models is another important parameter, as shorter digestion times are needed when only intestinal digestion is performed. The intestine is furthermore the primary site of absorption for macronutrients, micronutrients and active pharmaceutical ingredients (API), thus making it inherently important, as the aqueous solubility in intestinal fluid of APIs has been shown to correlate with the in vivo bioavailability. ${ }^{29,30}$ The incorporation of a gastric compartment to the intestinal in vitro lipoly- sis models has, however, also been hampered by the lack of

\footnotetext{
${ }^{a}$ Department of Pharmacy, Faculty of Health and Medical Sciences, University of Copenhagen, Copenhagen, Denmark.E-mail: anette.mullertz@sund.ku.dk

${ }^{b}$ Bioneer:Farma, University of Copenhagen, Copenhagen, Denmark

${ }^{c} N e s t l e ́$ Research Center, Lausanne, Switzerland
} 
availability of lipases with similar digestive properties as HGL. HGL is an acid stable lipase active in the pH-range 2-8 with pH-optima between 4-6 depending on the substrate. ${ }^{31-33}$ Its activity throughout a broad $\mathrm{pH}$-range makes it unique compared to pancreatic lipases, only displaying activity above $\mathrm{pH}$ 4.5. ${ }^{2,34}$ HGL does furthermore display a higher specific activity towards short chain (SC)- and medium chain (MC)-TAGs than long chain (LC)-TAGs. ${ }^{32,35}$ The catalytic affinity of HGL is solely towards FAs situated at the terminal carbons on the glycerol backbone, and primarily the sn-3 position. ${ }^{35}$ Sampling HGL from humans is difficult for ethical reasons and its natural low abundance compared to the pancreatic enzymes. ${ }^{1,2}$ Thus alternative lipases have been explored as substitutes for HGL in in vitro models.

A number of microbial lipases such as Rhizopus oryzae lipase (ROL) and Candida antarctica lipase A (CALA) have been used to mimic gastric digestion in vitro. ${ }^{27,36,37}$ ROL is facilitating digestion at the sn- 1 and sn-3 position (as does HGL), but HGL has a preference towards the sn-3 position whereas ROL shows a higher affinity towards the sn-1 position. ${ }^{35,36,38}$ ROL is commonly used in more complex in vitro digestion models, such as the TNO gastrointestinal model (TIM) and the Dynamic Gastric Model (DGM)..$^{27,39-42}$ The digestion products resulting from a complex dietary product, e.g. an infant formula, following digestion catalyzed by ROL have, to the best of the authors knowledge, never been compared with the digestion catalyzed by HGL. Thus ROL's utility as a substitute for HGL in in vitro digestion models is still to be validated. CALA has also been used for in vitro gastric digestion, since it has been shown to have a comparable activity-pH profile as

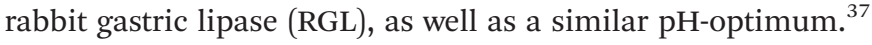
The digestion catalyzed by CALA has, however, previously been shown to occur predominantly on the sn-2 position, which HGL has no affinity for. ${ }^{35,38}$ This is particularly problematic, if the digestion model is simulating both gastric and intestinal compartments, since pancreatic lipase is sn-1 and sn-3 specific. ${ }^{35}$ Thus an overestimation of TAG liberated of all three fatty acids (FA) might occur, which would not take place in vivo where 2-monoglycerides are readily absorbed. ${ }^{21,43,44}$ Animal derived lipases, such as dog gastric lipase (DGL) and RGL have also been used for in vitro gastric digestion. ${ }^{45-48}$ DGL shows a higher activity towards LC-TAG than SC-TAG. ${ }^{45}$ This is a problem when digesting complex meals or drug delivery systems containing TAGs with FAs of varying chain lengths and DGL is therefore not suitable as a surrogate for HGL. Different studies with RGL have demonstrated, that RGL seems like a sensible replacement for HGL, as the rate of digestion has been shown to be comparable when using these two lipases. ${ }^{47}$ RGL does, however, have a lower specific activity than HGL. ${ }^{45,47}$ Studies comparing digestion products after lipolysis catalyzed by RGL and HGL have investigated the formation of monoacylglycerides (MAG) and diacylglycerides (DAG) from a solid meal. ${ }^{47}$ However, the relative preference of RGL for hydrolysis of FAs with different chain lengths has not been compared with HGL and might indeed play a crucial role in replicating nutrient update (in the infant for example), but also for solubilization of poorly water soluble micronutrients or APIs. ${ }^{49,50}$

The shortcomings of the above mentioned animal and microbial lipases, with regard to digestive properties compared to HGL, have lead to the expression of recombinant human gastric lipase (rHGL) in different cell-lines e.g. yeast, insect and tobacco plant cells. ${ }^{4,51,52}$ The digestive properties of these recombinant lipases have not been thoroughly investigated, but all recombinant lipases showed a lower specific activity than HGL. ${ }^{4,51,52}$ A novel rHGL has, however, recently been expressed in human embryonic kidney cells. ${ }^{53}$ This has ensured a similar structure and glycosylation of the protein, which has resulted in an enzyme with the same specific activity as HGL. ${ }^{53}$

Given the recent availability of rHGL, and the incomplete knowledge on RGL, it would seem critical to compare their digestive properties to ROL which is used in several established models. Thorough investigation of the digestive properties of these three lipases with regard to the nature and extent of the FAs released would assist in identifying a suitable gastric lipase for in vitro gastric digestion with similar digestive properties as HGL. This is of major importance in the development of predictive in vitro digestion models for both the food and pharmaceutical industry. For this purpose infant formulas (IF) were chosen as the model substrate given the importance of HGL in infant digestion. ${ }^{12,13}$ In addition, there are few in vitro studies that aim to mimic the biochemical environment of the infant stomach during digestion. Infants suffer from developmental digestive immaturity which affects proton pump activity (i.e. gastric $\mathrm{pH}$ in the fed state) and bile salt speciation/reflux. ${ }^{11,54-58}$ Clinical studies have examined gastric aspirates from infants fed with different IFs providing excellent data on the type and extent of FA released during digestion. ${ }^{10,11}$ Hence the clinical data on HGL's catalytic properties are available in literature and could thus provide the basis for a comparison with the FA release during in vitro lipolysis of IFs by different commercially available lipases. A key question discussed in these studies, and paediatric nutrition in general, is what is the role/extent of MC-FA release during gastric digestion. Therefore, the present study sets out to investigate the release of FAs during lipolysis of two IF; one containing predominantly LC-TAGs and one enriched with MC-TAG by three potential gastric lipase substitutes ROL, RGL and the novel rHGL. The qualitative release of FAs was compared to previously published clinical data during gastric digestion of comparable IFs. ${ }^{10}$

\section{Materials and methods}

\section{Materials}

Sodium taurocholate, sodium chloride, hydrochloric acid, sodium hydroxide, potassium hydroxide, porcine pepsin, maleic acid, tris(hydroxymethyl)-aminomethane, porcine pancreatin, rhizopus oryzae lipase were purchased from SigmaAldrich (St Louis, MO, USA). Rabbit gastric lipase was kindly 
Table 1 Composition of major fatty acids in the infant formulas used as substrates. The values listed are the amounts added to each digestion and were quantified by $\mathrm{GC}$

\begin{tabular}{lcc}
\hline Fatty acids & MC-IF $(\mu \mathrm{mol} \mathrm{FA})$ & LC-IF $(\mu \mathrm{mol} \mathrm{FA})$ \\
\hline C8:0 & $184.8 \pm 0.9$ & $11.9 \pm 0.1$ \\
C10:0 & $142.5 \pm 0.3$ & $9.0 \pm 0.0$ \\
C12:0 & 0 & $56.0 \pm 0.1$ \\
C14:0 & 0 & $23.0 \pm 0.0$ \\
C16:0 & $73.1 \pm 0.2$ & $109.1 \pm 0.1$ \\
C18:0 & $10.1 \pm 0.1$ & $15.7 \pm 0.0$ \\
C18:1 n9 cis & $170.2 \pm 0.3$ & $242.3 \pm 0.0$ \\
C18:2 n6 & $89.1 \pm 0.2$ & $92.9 \pm 0.1$ \\
C18:3 n6 & $12.2 \pm 0.2$ & $11.5 \pm 0.1$ \\
Total FAs available for digestion & 682 & 571 \\
& &
\end{tabular}

donated by Germe S.A. (Marseilles, France). The purified rHGL was donated by Bioneer A/S (Hoersholm, Denmark). The IFs used were PRENAN Stage 1 \& 2 obtained from Nestlé (Lausanne, Switzerland). PRENAN stage 1 (MC-IF) is enriched with MC-TAG and PRENAN stage 2 (LC-IF) is a predominantly LC-TAG formula (Table 1). The lipid composition of the infant formulas were quantified by gas chromatography (GC) as explained below in the method section. The methylated FAs (FAME) standards used for GC were all obtained from $\mathrm{Nu}$ Chek-Prep (Elysian, MN, USA). All the water used in this study was of purified Milli Q quality. All other solvents and reagents were of analytical grade or higher.

\section{In vitro gastrointestinal digestion}

The gastrointestinal in vitro lipolysis experiments were carried out according to Wooster et al. with minor modifications. ${ }^{59}$ The digestions were conducted in thermostated glass vessels $\left(37{ }^{\circ} \mathrm{C}\right)$ in either a pH-STAT setup controlled by a TIM 856 biburette pH-STAT (Radiometer Analytical, France) at pH 5.5 or at a dynamic pH-gradient from 6 to 4 during $60 \mathrm{~min}$ by a continuous addition of $\mathrm{HCl}$ at a constant rate via a Harvard syringe pump (Harvard Apparatus, MA, USA). The gastric digestions were conducted in $4.25 \mathrm{ml}$ simulated gastric fluid (Table 2) to which $2 \mathrm{ml}$ of MC-IF or LC-IF were added (only MC-IF was used as substrate for the dynamic $\mathrm{pH}$ experiments). The digestion was initiated by adding 18 tributyrin units per $\mathrm{ml}$ ((TBU) activity determined at pH 5.4 according to Armand

Table 2 Composition of the medium used for gastric digestions before dilution with IF

\begin{tabular}{lc}
\hline Medium composition & Concentration \\
\hline NaTC & $80 \mu \mathrm{M}$ \\
NaCl & $68 \mathrm{mM}$ \\
Tris & $2 \mathrm{mM}$ \\
Maleic acid & $2 \mathrm{mM}$ \\
Phospholipid & $20 \mu \mathrm{M}$ \\
\hline
\end{tabular}

Added to initiate digestion

Pepsin

Lipase (ROL, RGL or rHGL)
$450 \mathrm{U} \mathrm{ml}^{-1}$ $18 \mathrm{TBU} \mathrm{ml}^{-1}$
Table 3 Final composition of the simulated intestinal fluid after dilution by gastric digesta

\begin{tabular}{lc}
\hline Medium composition & Concentration \\
\hline NaTC & $2 \mathrm{mM}$ \\
NaCl & $150 \mathrm{mM}$ \\
Tris & $2 \mathrm{mM}$ \\
Maleic acid & $2 \mathrm{mM}$ \\
Phospholipid & $0.18 \mathrm{mM}$
\end{tabular}

et al. $1994^{7}$ ) of either lipase (ROL, RGL or rHGL) and 450 $\mathrm{U} \mathrm{ml}^{-1}$ pepsin, based on enzyme levels reported in vivo. ${ }^{2,3,7-9,60}$ Samples were aspirated after 0, 5, 15, 30, 45 and $60 \mathrm{~min}$ and digestion was stopped by a liquid-liquid extraction of lipids with chloroform: methanol $(2: 1)$. The remaining digesta were diluted 1.7 times with a concentrated intestinal medium to obtain an environment comparable to the small intestine of a fed infant (Table 3 ) ${ }^{55-58}$ Digestions under intestinal conditions were conducted at $\mathrm{pH} 6.5$ with and without the presence of pancreatin, to elucidate the digestive capacity of the gastric lipases under intestinal conditions and to ensure that the IFs were completely digestible (66\% release of FAs). Samples were taken at $90 \mathrm{~min}$ (60 min of gastric digestion + $30 \mathrm{~min}$ of intestinal digestion) for the intestinal digestions without pancreatin and after $180 \mathrm{~min}$ (60 min of gastric digestion +120 min of intestinal digestion) for intestinal digestion in the presence of pancreatin (300 USP units per $\mathrm{ml}$ ).

\section{Fatty acid analyses}

Fatty acid (FA) analyses were performed on the extracted lipids at all time points, according to Destaillats et al. $2007 .{ }^{61}$ The organic phase (chloroform:methanol 2:1) containing the lipids was transferred to glass test tubes and evaporated to dryness under nitrogen. The dried lipids were redissolved in hexane $(1 \mathrm{ml})$ and divided into two fractions of $450 \mu \mathrm{l}$, one for acid methylation and one for alkaline methylation, to determine all the FAs present in the digesta, including FFA, esters, alk-1-enyl ethers, amides and glycosides, and to determine the fraction of FAs bound to glycerol, respectively. ${ }^{62}$

\section{Acid methylation}

Internal standard (100 $\mu \mathrm{l}, 0.5 \mathrm{mg} \mathrm{m}^{-1}$ TAG (C13) + fatty acid methyl esters (FAME) of C11 in hexane), $2 \mathrm{ml}$ of methanol, $2 \mathrm{ml}$ of methanolic acid ( $3 \mathrm{~N} \mathrm{HCl}$ in methanol) and $450 \mu \mathrm{l}$ hexane were added to the $450 \mu \mathrm{l}$ lipid solution. The test tubes were vortexed vigorously for $1 \mathrm{~min}$ before incubation for $60 \mathrm{~min}$ at $80^{\circ} \mathrm{C}$ and subsequently allowed to cool to room temperature. After samples had reached room temperature, $2 \mathrm{ml}$ of water was added to seize the methylation and samples were vortexed for 2 min followed by centrifugation in a Thermo Scientific Megafuge centrifuge (Osterode, Germany) (5 min at $1500 \mathrm{rpm} 21^{\circ} \mathrm{C}$ ). The upper phase was collected for GC.

\section{Alkaline methylation}

The $450 \mu$ l lipid solution was evaporated to dryness under nitrogen. $100 \mu \mathrm{l}$ of internal standard $\left(0.5 \mathrm{mg} \mathrm{ml}^{-1} \mathrm{TAG}(\mathrm{c} 13)+\right.$ 
FAME c11 in hexane) and $200 \mu \mathrm{l} 2 \mathrm{M} \mathrm{KOH}$ in methanol were added to the test tube and samples were vortexed for $3.5 \mathrm{~min}$. The reaction was then seized by the addition of $400 \mu \mathrm{l} 1 \mathrm{M} \mathrm{HCL}$ and samples were briefly vortexed. $900 \mu \mathrm{l}$ hexane was added and samples were vortexed for 2 min followed by centrifugation in a Megafuge centrifuge (Osterode, Germany) (5 min at $1500 \mathrm{rpm} 21^{\circ} \mathrm{C}$ ). The upper phase was collected for GC.

The release of FAs during digestion was measured as the difference between all the FAs present (acid methylation) and FAs bound to glycerol (alkaline methylation).

\section{Gas chromatography}

Analyses of the FAMEs were conducted on a 7890 Agilent gas chromatograph equipped with a flame ionization detector (Agilent Technologies, Palo Alto, CA, USA). The column was a fused-silica BPX-70 $(10 \mathrm{~m} \times 0.1 \mathrm{~mm}$ I.D., $0.2 \mu \mathrm{m}$ film thickness; SGE, Melbourne, Australia). The settings of the instrument were adopted from Destaillats et al. 2007 and each sample had a run time of 4.9 min. ${ }^{61}$

\section{Droplet size}

The droplet size of MC- and LC-IFs during dispersion (0 min digestion) in gastric medium was measured using a Mastersizer 3000 equipped with a Hydro SM from Malvern Instruments (Southborough, MA, USA). The laser specifications of the two lasers are $4 \mathrm{~mW} 632.8 \mathrm{~nm}$ and $10 \mathrm{~mW}$ $470 \mathrm{~nm}$. The laser obscuration was approximately $5 \%$ and a refractive index of 1.456 was used. The droplet size of the LC-IF after 60 min gastric digestion by any of the three lipases was also investigated.

\section{Droplet morphology}

The droplet morphology of LC-IF before and after $60 \mathrm{~min}$ digestion by either ROL, RGL or rHGL was investigated by scanning electron microscopy (SEM). Sample preparation was done according to the procedure proposed by Pafumi et al. $2002 .^{63}$ Emulsions were collected at the desired time point and mixed at $1: 1$ with $1 \%$ Osmium tetroxide solution at room temperature. The mixture was carefully shaken and few drops were put on a microscope cover glass. Fixation was performed overnight in a moist chamber at room temperature. The fixed emulsion was subsequently gently washed with distilled water before being dried using a filter paper and a desiccator. Samples were coated with a $5 \mathrm{~nm}$ gold layer before being observed using a Quanta 200 FEG Scanning Electron Microscope (FEI Company, The Netherlands) at $10 \mathrm{kV}$ in high vacuum mode. The droplet morphology of MC-IF could not be captured by the present sample preparation method, since Osmium tetroxide only is able to react with double bonds and saturated FAs are abundant in MC-IF (Table 1).

\section{Results}

It was intended to investigate if the experimental setup, $\mathrm{pH}-\mathrm{STAT}$ or the more physiological relevant dynamic $\mathrm{pH}$

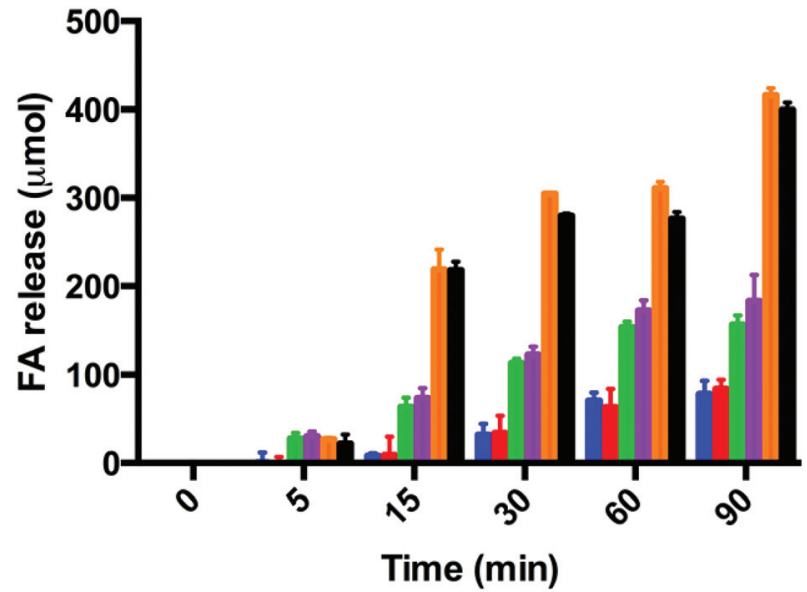

Fig. 1 Absolute release of total FAs during digestion of MC-IF by ROL, $\mathrm{RGL}$ and $\mathrm{rHGL}$, in the $\mathrm{pH}-\mathrm{STAT}$ setup and the dynamic $\mathrm{pH}$-gradient setup. $\square=\mathrm{pH}$-STAT RGL, $\square=\mathrm{pH}$-dynamic RGL, $\square=\mathrm{pH}-\mathrm{STAT}$ rHGL, $=\mathrm{pH}$-dynamic rHGL, $\square=\mathrm{pH}-\mathrm{STAT} \mathrm{ROL}, \mathbf{\square}=\mathrm{pH}$-dynamic ROL.

gradient affected the degree of digestion during lipolysis of MC-IF by either of the three gastric lipases. However, the results obtained by the two models were almost superimposable (Fig. 1). This indicates that the three tested lipases are displaying approximately the same activity in the $\mathrm{pH}$-interval 4 to 6 or that they in average over the course of the experiment display the same activity in the two setups. Thus digestion of LC-IF digestion was only carried out by the pH-stat method.

\section{Comparison of fatty acids released during digestion of MC-IF catalyzed by ROL, RGL and rHGL}

FA release catalyzed by ROL, RGL and rHGL during digestion of MC-IF is shown in Fig. 2a and b. With regard to digestion by ROL, a lag phase of at least 5 min was seen, where only $4 \%$ of the total FAs available for digestion were liberated (Fig. 2a). At $15 \mathrm{~min}$ of digestion $32 \%$ of the FAs were liberated. The rate of lipolysis then leveled off and $45 \%$ and $46 \%$ of the FAs were liberated after 30 and $60 \mathrm{~min}$, respectively. After $30 \mathrm{~min}$ of intestinal incubation (time point $90 \mathrm{~min}$ ), without addition of pancreatin, $61 \%$ of the FAs had been hydrolyzed by ROL. It can furthermore be observed in Fig. $2 b$ that ROL appears insensitive of FA chain length and abundance, since 50-75\% of all FAs were released relative to their initial composition. The extent of digestion catalyzed by RGL was modest, compared to ROL; only $0 \%, 1 \%, 5 \%, 10 \%$ and $11 \%$ of the total FAs were liberated after $5 \mathrm{~min}, 15 \mathrm{~min}, 30 \mathrm{~min}, 60 \mathrm{~min}$ and $90 \mathrm{~min}$, respectively (Fig. 2a). RGL released only C8:0 and C10:0 under both gastric and intestinal conditions (Fig. 2b), indicating that RGL is highly specific toward hydrolysis of medium chain FA. During the digestion catalyzed by rHGL, $4 \%, 9 \%, 16 \% 22 \%$ and $23 \%$ of the FAs were liberated after $5 \mathrm{~min}, 15 \mathrm{~min}, 30 \mathrm{~min}, 60 \mathrm{~min}$ and $90 \mathrm{~min}$, respectively (Fig. 2a). As for RGL, also rHGL showed a certain specificity towards hydrolysis of medium chain FA, i.e. C8:0 and C10:0 were released preferentially, but the release of FAs C16:0 to 

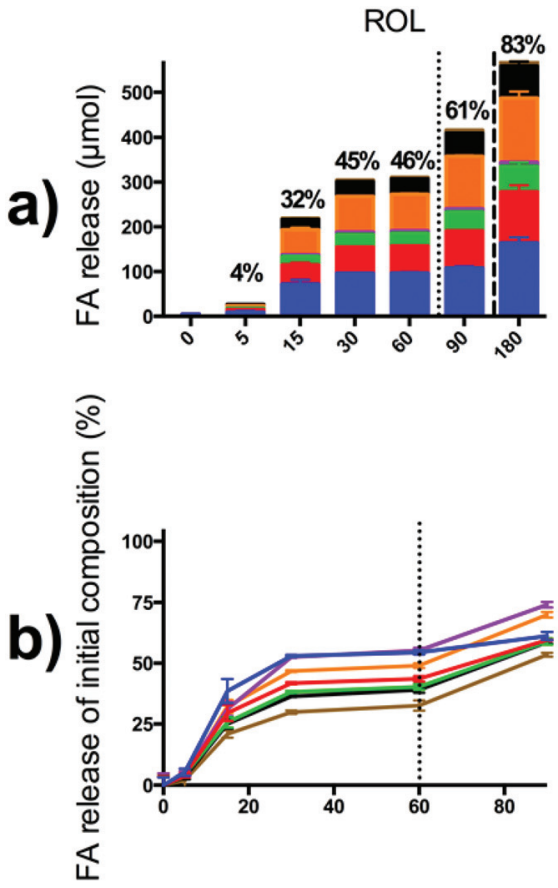
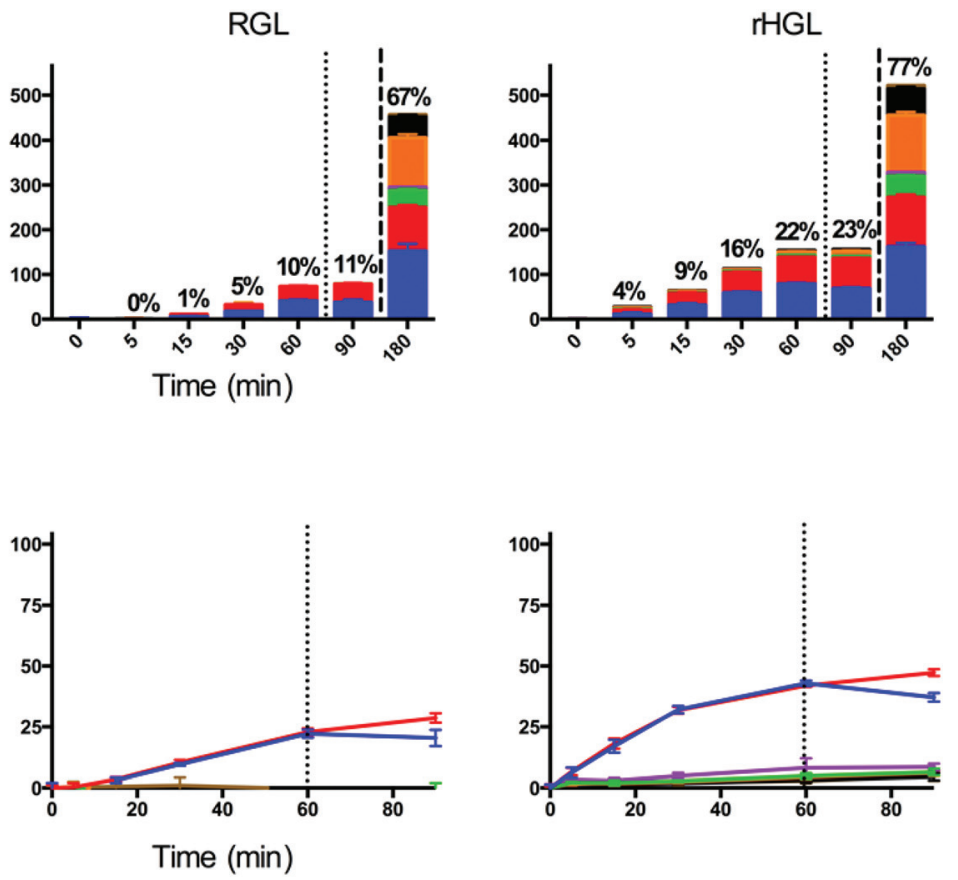

Fig. 2 (a) Absolute release of FAs during digestion of MC-IF by ROL, RGL and rHGL. The percentages indicate the percentage of FAs liberated relative to the total amount present in MC-IF. (b) Release of FA relative to the initial composition in the IF during digestion of MC-IF catalyzed by ROL, RGL and rHGL. The dotted lines indicate change from gastric to intestinal conditions. The dashed lines indicates digestion in the presence of pancreatin. $\square=\mathrm{C} 8: 0, \square=\mathrm{C} 10: 0, \square=\mathrm{C} 16: 0, \square=\mathrm{C} 18: 0, \square=\mathrm{C} 18: 1 \mathrm{n} 9 \mathrm{cis}, \boldsymbol{\square}=\mathrm{C} 18: 2 \mathrm{n} 6, \square=\mathrm{C} 18: 3 \mathrm{n} 6$

C18:2n6 was also evident (Fig. 2b). Furthermore, it is worth noticing that MC-IF was fully digestible ( $>66 \%$ FA released, as 2-monoglycerides are readily absorbed in vivo) in the presence of pancreatin (180 $\mathrm{min}$ ) and either of the tested lipases. Thus MC-IF seems to be fully digestible under in vitro conditions simulating the GI-tract of an infant and could therefore also be believed to be fully digestible in the infant in vivo, securing optimal nutritional uptake.

\section{Comparison of fatty acids released during digestion of LC-IF catalyzed by ROL, RGL and rHGL}

FA release during digestion of LC-IF by ROL, RGL and rHGL is shown in Fig. 3a and b. Using ROL as gastric lipase, the initial digestion occurred very rapidly; $33 \%$ of FAs were hydrolyzed after $5 \mathrm{~min}$. The following digestion rate was slower and 38\%, $42 \% 49 \%$ and $66 \%$ of the FAs were hydrolyzed after $15 \mathrm{~min}$, $30 \mathrm{~min}, 60 \mathrm{~min}$ and $90 \mathrm{~min}$, respectively (Fig. 3a). As observed for MC-IF, ROL seems to be insensitive to FA chain length or abundance, as $50-80 \%$ of all FAs were released relative to their initial composition (Fig. 3b). LC-IF digestion by RGL liberated $6 \%, 11 \%, 13 \%, 18 \%$ and $23 \%$ after $5 \mathrm{~min}, 15 \mathrm{~min}, 30 \mathrm{~min}$, $60 \mathrm{~min}$ and $90 \mathrm{~min}$, respectively (Fig. 3a). Again RGL proved to be sensitive to FA chain length and showed a clear preference for FAs with shorter chain lengths. The rank order of the extent of digestion relative to the initial FA composition was C8:0 > C10:0 > C12:0 > C14:0 > C16:0, with the rest of the FAs being digested at comparable rates (Fig. 3b). Lauric acid (C12:0) had the highest total FA release $(37 \mu \mathrm{mol})$, despite oleic acid (C18:1) being the most abundant FA in LC-IF. rHGL hydrolyzed $6 \%, 12 \%, 16 \%, 21 \%$ and $24 \%$ after $5 \mathrm{~min}, 15 \mathrm{~min}$, $30 \mathrm{~min}, 60 \mathrm{~min}$ and $90 \mathrm{~min}$ of digestion, respectively. The FA chain length did again appear to matter and the rank order of the digestion rates with regard to FAs released relative to the initial composition was $\mathrm{C8:0}>\mathrm{C} 10: 0>\mathrm{C} 12: 0$, the rest of the FAs were liberated at comparable rates (Fig. 3b). Oleic acid was, however, the most released FA in absolute numbers (48 $\mathrm{mmol}$ ) (Fig. 3a).

LC-IF did appear to be fully digestible after addition of pancreatic enzymes for ROL and rHGL (>66\% FA released). Only $64 \%$ of the FAs were released during intestinal digestion in the presence of pancreatin and RGL, which however, was very close to full digestibility. Thus LC-IF does also seem to be fully digestible under experimental conditions simulating the GItract of an infant. This indicates, that all fat in the formula is available for absorption in vivo.

\section{Droplet morphology}

The micrographs of LC-IF before and after gastric digestion by ROL, RGL and rHGL are shown in Fig. 4a-d. The micrograph in Fig. 4a shows the oil globules of LC-IF prior to initiation of digestion. The globules appeared as uniform spherical particles with a smooth surface/interface. Aggregation seems to be limited, which is supported by the droplet size data shown in Table 4 , where it can be seen that $90 \%$ of the droplets were smaller than $1.0 \mu \mathrm{m}$ (Dx (90)) and 50\% were smaller than $0.5 \mu \mathrm{m}$ (Dx (50)). The morphological change of the oil globules 
ROL
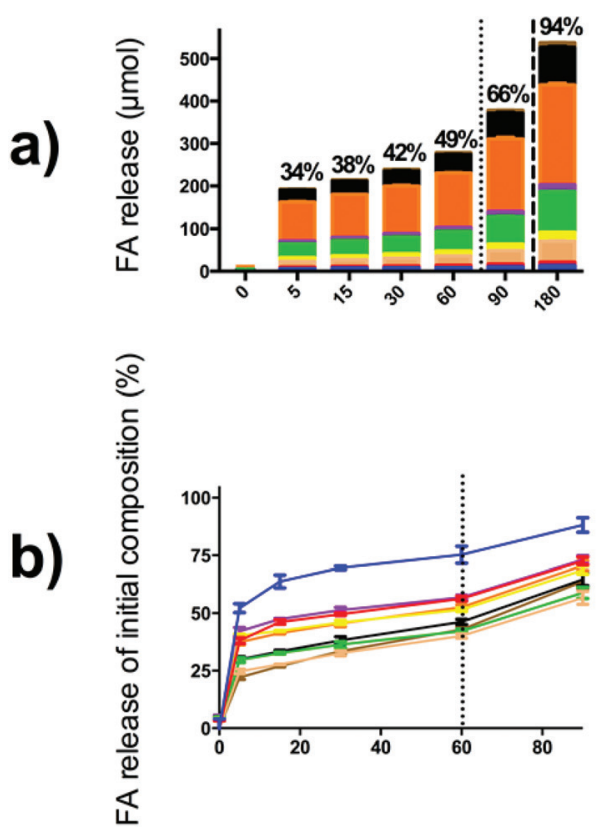

RGL
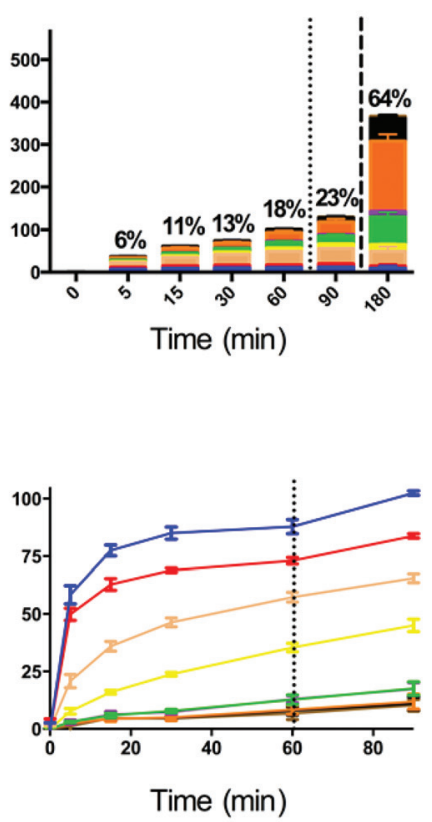

rHGL
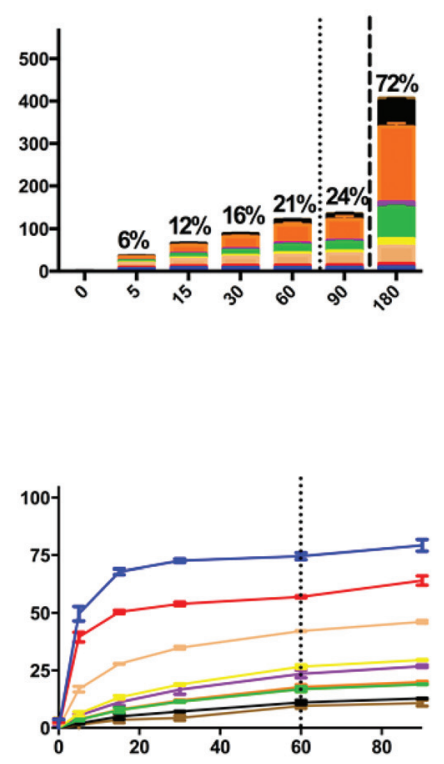

Fig. 3 (a) Absolute release of FAs during digestion of LC-IF by ROL, RGL and rHGL. The percentages indicate the amount of FAs liberated relative to the total amount present in LC-IF. (b) Release of FA relative to the initial composition in the IF during digestion of LC-IF catalyzed by ROL, RGL and rHGL. The dotted lines indicate change from gastric to intestinal conditions. The dashed lines indicate intestinal digestion in the presence of pancreatin. $\square=\mathrm{C} 8: 0, \square=\mathrm{C} 10: 0, \square=\mathrm{C} 12: 0, \square=\mathrm{C} 14: 0, \square=\mathrm{C} 16: 0, \square=\mathrm{C} 18: 0, \square=\mathrm{C} 18: 1 \mathrm{n} 9 \mathrm{cis}, \mathbf{\square}=\mathrm{C} 18: 2 \mathrm{n} 6, \square=\mathrm{C} 18: 3 \mathrm{n} 6$.
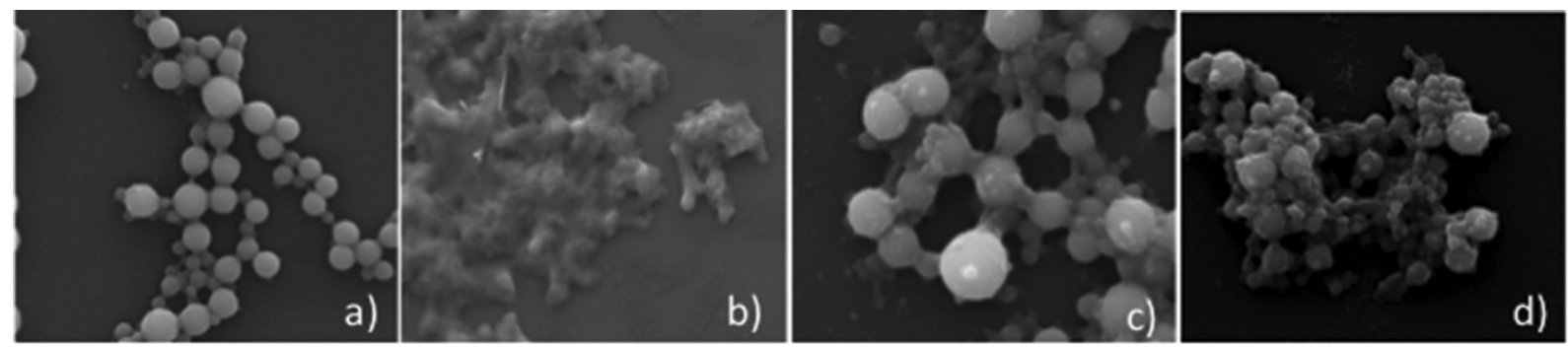

Fig. 4 SEM micrographs of a: LC-IF prior to digestion, b: LC-IF after 60 min gastric digestion by ROL, C: LC-IF following 60 min gastric digestion by RGL, and d: LC-IF following 60 min gastric digestion by $r H G L$.

Table 4 Droplet size data of MC-IF and LC-IF prior to digestion and after 60 min gastric digestion of LC-IF by ROL, RGL and rHGL, determined by laser diffraction

\begin{tabular}{|c|c|c|c|c|c|c|}
\hline Sample name & Digestion time (min) & $D[3,2](\mu \mathrm{m})$ & $D[4,3](\mu \mathrm{m})$ & $\mathrm{Dx}(10)(\mu \mathrm{m})$ & $\mathrm{Dx}(50)(\mu \mathrm{m})$ & $\operatorname{Dx}(90)(\mu \mathrm{m})$ \\
\hline LC-IF & 0 & $0.4 \pm 0.0$ & $0.6 \pm 0.0$ & $0.2 \pm 0.0$ & $0.5 \pm 0.0$ & $1.0 \pm 0.1$ \\
\hline LC-IF rHGL & 60 & $0.2 \pm 0.0$ & $1.0 \pm 0.0$ & $0.1 \pm 0.0$ & $0.4 \pm 0.0$ & $2.7 \pm 0.0$ \\
\hline
\end{tabular}

after 60 min of digestion by ROL can be seen in Fig. 4b. It is evident that the integrity of the globules has been disrupted and their spherical shape was almost completely lost. The surface was no longer smooth. Furthermore, there were indications of extensive aggregation, which again was supported by the droplet size data in Table 4 , where an almost 10 -fold increase in Dx (50) to $4.2 \mu \mathrm{m}$ was observed. A representative example of the appearance of the oil globules after $60 \mathrm{~min}$ digestion by RGL can be seen in Fig. 4c. The spherical shape of the globules was intact, however small spherical protrusions were present on the surface of the droplets. The droplet size measurements showed an increase in Dx (50) and Dx (90) to 
$0.5 \mu \mathrm{m}$ and $12.7 \mu \mathrm{m}$, respectively, following RGL digestion. The same trend was found after $60 \mathrm{~min}$ of digestion by rHGL, where globule integrity appeared uncompromised and there were many small spherical protrusions present at the surface of the droplets. The droplet size was altered modestly to $0.4 \mu \mathrm{m}$ and $2.7 \mu \mathrm{m}$ for Dx (50) and Dx (90), respectively.

\section{Discussion}

\section{Comparison of in vitro digestion of MC-IF by ROL, RGL and rHGL}

Great differences were observed between the digestive properties of the three lipases tested (Fig. 2a and b). The differences were not only evident in the total extent of digestion, but also in the preference for hydrolysis of FAs of different chain length. The extent of digestion catalyzed by ROL ( $46 \%$ after 60 min gastric digestion) was considerably higher than for the other two lipases $(10 \%$ and $22 \%$ for RGL and rHGL, respectively). This is remarkable since the same standardized amount of lipase activity (18 TBU ml $\mathrm{Tl}^{-1}$ ) was present during the experiment for all three lipases. This shows that ROL has different digestive properties compared to the other two lipases. The extent of digestion by ROL was also significantly higher than what has been reported for HGL in vivo, where $10-45 \%$ of the total hydrolysis occurred via gastric digestion. ${ }^{1}$ Total hydrolysis of a TAG corresponds to $66 \%$ liberation of the FAs present, hence $46 \%$ FAs released by ROL corresponds to $69 \%$ of the total hydrolysis. ${ }^{43,44}$ Furthermore, ROL catalyzed extensive digestion under intestinal conditions where hydrolysis increased from 46 to $61 \%$ of all FAs present (92\% of the total hydrolysis). In addition it was apparent that ROL digested FAs of all chain lengths at approximately the same rate relative to their initial composition in the IF. This is in contrast with in vivo data for HGL by Hamosh et al. on the digestion of IFs with a similar lipid composition. ${ }^{10}$ In their study, gastric samples were aspirated $15 \mathrm{~min}$ after feeding of IFs to infants. ${ }^{10}$ It was shown that MC-FAs were clearly the preferred substrate for HGL catalyzed hydrolysis when MC-TAG was present in combination with LC-TAGs, even though small amounts of LC-FAs were also released. In another study by Roman et al. samples were aspirated from the stomach after dosing of an MC-TAG enriched IF to infants. ${ }^{11}$ The authors did not see a clear preference for MC-TAGs as a substrate for HGL. ${ }^{11}$ However, they did not sample until 90 min after dosing and were therefore not able to see an initial preference for MC-TAG.

Thus ROL's lack of specificity with regard to fatty acid chain length does not replicate the action of HGL in vivo. This is indeed a problem if one wants to utilize gastrointestinal digestion models to evaluate if newly developed nutritional products or drug delivery systems are substrates for gastric digestion. ROL would then misleadingly show that all FAs are released at more or less the same rate. The non-specific digestive pattern of ROL could potentially also be a problem for the assessment of micronutrient or API solubilization by colloidal structures, as the solubilizing capacity of digestion products following hydrolysis of MC-TAGs and LC-TAGs is different. ${ }^{49,50}$ Thus based on the current study, ROL does not seem to replicate the digestive properties of HGL and is therefore not an appropriate surrogate for HGL in gastric in vitro lipolysis models.

RGL and rHGL both showed a higher affinity for the MC-FAs during digestion of MC-IF, which is a similar preference to what has been previously reported in vivo. ${ }^{10} \mathrm{RGL}$ did, however, only release MC-FAs, whereas rHGL predominantly released MC-FAs but also LC-FAs to some degree. Only very modest digestion occurred under intestinal conditions for both lipases (approximately 1\%). This is another important difference between ROL and the other two lipases. However, it is difficult to determine what would occur in vivo, due to the inherent presence of pancreatic lipases. The in vitro gastric digestion by rHGL seemed to replicate the digestive properties of HGL in vivo both with regards to total extent of digestion, but also fatty acid chain length preference. ${ }^{10}$ RGL also seems to be an acceptable candidate as a surrogate for HGL, since the extent of digestion is within the range reported in vivo and has a similar preference for MC-FA. It would, however, (misleadingly) show that LC-TAGs are not a substrate if present in the same blend as MC-TAGs and should therefore be used with care. An explanation for RGL's selective preference for MC-TAGs could possibly be found in the fact that rabbit's milk is rich in MC-TAGs (approximately 75\% C8 and C10). ${ }^{64}$ It does therefore seem plausible from an evolutionary point of view that MC-TAGs are excellent substrates for RGL. Thus a comparison between the current study and previously reported in vivo data by Hamosh et al., ${ }^{10}$ revealed that a rank order could be constructed indicating which lipase replicated the digestive pattern of HGL the best: rHGL > RGL > ROL.

\section{Comparison of in vitro digestion of LC-IF by ROL, RGL and rHGL}

The results in Fig. 3a and b showed substantial differences between the digestive properties of the three gastric lipases. ROL again showed a higher extent of gastric hydrolysis (49\% release of the total FAs present in the IF after 60 min digestion) compared to the two other lipases which hydrolysed $23 \%$ and $24 \%$ for RGL and rHGL, respectively. The chain length specificity/preference also varied between the three different lipases. ROL again seemed to hydrolyze FAs more or less indiscriminate of chain length compared to the other two lipases, which displayed clear preferences towards MC FAs. The insensitivity of ROL to FA chain length is not in line with what had previously been reported for HGL during the in vivo digestion of a LC-IF, where C8:0 and C10:0 were the first site of hydrolysis followed by C18:1. ${ }^{10}$ In our current study, RGL showed a clear preference towards MC-TAGs, C12:0 was the most released FA in absolute numbers and not C18:1. In vivo digestion of two commercial infant formulas with similar FA compositions suggest that whilst considerable amounts of MC-FAs are released by HGL action in the stomach, long chain FAs, specifically C18:1 and C18:2, are the largest fraction of FA in 
the stomach contents. ${ }^{10}$ The balance between amount of C12 and C18:1 FA released observed in vivo by Hamosh et al. were, however, replicated during the digestion of LC-IF catalyzed by rHGL, since rHGL showed a preference towards MC-TAGs, but C18:1 was the most released FA. ${ }^{10}$ Thus if one should construct the rank order at which the three different gastric lipases replicated the digestive properties of HGL it would again be rHGL > RGL $>$ ROL as observed during the digestion of MC-IF.

The differences in the digestive pattern of the three tested lipases do not only affect the total extent of digestion and the relative preference for different FA chain length, but also the morphology of the resulting oil globules, as observed from the SEM micrographs in Fig. 4a-d and the droplet size measurements in Table 4. The extensive gastric digestion occurring during ROL hydrolysis had completely disrupted the spherical shape of the oil globules, and droplet aggregation seemed evident. This was also confirmed by the droplet size data were Dx (50) Dx (90) were $4.2 \mu \mathrm{m}$ and $7.0 \mu \mathrm{m}$, respectively, which was considerably higher than the droplet size prior to digestion. The oil globules after gastric digestion by RGL and rHGL were very similar in appearance and the spherical shape was still intact. However, unusual spherical protrusions were observed on the surface of the lipid droplets after gastric digestion by both RGL and rHGL. Such protrusions have only been reported in a few studies. They were initially discovered by Pafumi et al. during in vitro digestion of model emulsions and Gallier et al. recently observed similar structures during the in vivo digestion of bovine milk. ${ }^{63,65}$ Pafumi et al. explored the role these structures played in the inhibition of gastric lipolysis and reported that these structures are largely composed of free FAs $(73.7 \%$ long chain), phospholipids (15.6\%) and MAGS and DAGS (8.5\%), resulting from the digestive action of HGL. ${ }^{63}$ Pafumi et al. propose that the build-up of poorly water-soluble LC-FA at the interface inhibit the action of HGL by forming the lipidic protrusions which envelop HGL preventing it from accessing TAGs at the interface. ${ }^{63}$ The fact that RGL and rHGL replicate this behavior highlights the similarity in their surface behavior to HGL. The presence of extensive gastric hydrolysis in vitro by ROL suggests it has very different surface properties to HGL as it is not inhibited by the build-up of LC-FA digestion products.

Differences between RGL and rHGL were, however, indicated in the droplet size measurements in Table 4. The Dx (50) and Dx (90) were $0.54 \mu \mathrm{m}$ and $12.74 \mu \mathrm{m}$ following RGL digestion and $0.39 \mu \mathrm{m}$ and $2.7 \mu \mathrm{m}$ following rHGL digestion, respectively. This suggests that more extensive aggregation of the oil droplets occurred following digestion by RGL compared to rHGL. Thus, even though the extent of digestion was very similar between RGL and rHGL, the resulting digestion products had different propensities for droplet aggregation. This indicates that the choice of lipase does not only affect substrate specificity or regio- or stereo-specifity, but also the resulting droplet morphology. This could potentially be important for micronutrient and API absorption since the colloidal structures would differ depending on the lipase used. The rate of intestinal digestion might also be altered with the change of surface area available for hydrolysis. This was actually observed in the present study, the extent of intestinal digestion by pancreatin was $64 \%$ and $72 \%$ in the presence of RGL and rHGL, respectively. Thus aggregation of oil droplets following gastric digestion by RGL could explain the reduced extent of intestinal digestion compared to rHGL. It should, however, be noted that other factors than the surface area available for digestion, could influence the extent of intestinal digestion, such as the FA composition on the remaining TAGs and DAGS.

Overall when MC-IF and LC-IF were digested in the two compartment digestion model (containing gastric lipase and pancreatin) both formulas underwent complete digestion. Under human in vivo conditions complete digestion occurs at approximately $66.6 \%$ hydrolysis, i.e. the conversion of the TAG into a MAG and two FAs. ${ }^{66}$ Digestion of the two formulas in the presence of $\mathrm{RGL} /($ porcine)pancreatin resulted in $67 \%$ (MCT) and 64\% hydrolysis (LCT) whilst for $\mathrm{rHGL} /$ pancreatin resulted in 77 and $72 \%$ hydrolysis respectively. Interestingly, digestion by ROL/pancreatin resulted in 83 and 94\% hydrolysis of MC-IF and LC-IF, respectively, well above what would normally be expected in vivo. The higher than expected hydrolysis for ROL/pancreatin was quite surprising, but could possibly be attributed to acyl migration and/or reactivity at the sn- 2 position at elevated $\mathrm{pH}$-levels or in cooperation with other lipases, such as carboxylester hydrolase present in porcine pancreatin. ${ }^{67,68}$

\section{Conclusion}

The digestions of MC-IF and LC-IF by ROL, RGL and rHGL revealed that the lipases varied both with regard to the extent of digestion, FA affinity and morphology changes of oil globules during digestion. ROL showed a much higher extent of digestion than expected based on previous in vivo data both under gastric and intestinal conditions and was furthermore indiscriminative of FA chain length and abundance. RGL and rHGL both showed an extent of digestion within the expected range and they furthermore displayed a preference towards MC-TAGs. RGL did, however, only release MC-FAs during the digestion of MC-IF whereas rHGL predominantly hydrolyzed MC-TAGs, but also some LC-TAGs. Previously reported in vivo data states that MC-TAGs are preferentially hydrolyzed in vivo but also some digestion of LC-TAGs occur. A result replicated by rHGL, but not RGL. The digestion of LC-IF also revealed that rHGL seemed to replicate the digestive pattern observed in vivo with regard to FA specificity. Furthermore the formation of lumbs on the oil droplet surface during digestion catalyzed by HGL, as observed previously by Pafumi et al. and Gallier et al., was reproduced by RGL and rHGL in this present study. ${ }^{63,65}$ Hence RGL and rHGL seem to have similar surface properties as HGL. Thus, of the tested lipases, rHGL seems to be the best surrogate for HGL for in vitro digestion models.

\section{Acknowledgements}

The study was conducted as part of the Oral Biopharmaceutics Tools (ORBITO) project (http://www.orbitoproject.eu) funded 
by the Innovative Medicines Initiative (IMI) Joint Undertaking under Grant Agreement No. 115369. The study was furthermore conducted at Nestlé Research Center, Lausanne, Switzerland and was made possible through funding from Nestlé.

\section{References}

1 M. Armand, Curr. Opin. Clin. Nutr. Metab. Care, 2007, 10, 156-164.

2 F. Carriere, J. A. Barrowman, R. Verger and R. Laugier, Gastroenterology, 1993, 105, 876-888.

3 F. Carriere, C. Renou, V. Lopez, J. De Caro, F. Ferrato, H. Lengsfeld, A. De Caro, R. Laugier and R. Verger, Gastroenterology, 2000, 119, 949-960.

4 C. WickerPlanquart, S. Canaan, M. Riviere, L. Dupuis and R. Verger, Protein Eng., 1996, 9, 1225-1232.

5 H. Moreau, R. Laugier, Y. Gargouri, F. Ferrato and R. Verger, Gastroenterology, 1988, 95, 1221-1226.

6 S. J. Denigris, M. Hamosh, D. K. Kasbekar, T. C. Lee and P. Hamosh, Biochim. Biophys. Acta, 1988, 959, 38-45.

7 M. Armand, P. Borel, C. Dubois, M. Senft, J. Peyrot, J. Salducci, H. Lafont and D. Lairon, Am. J. Physiol., 1994, 266, G372-G381.

8 M. Armand, P. Borel, B. Pasquier, C. Dubois, M. Senft, M. Andre, J. Peyrot, J. Salducci and D. Lairon, Am. J. Physiol., 1996, 271, G172-G183.

9 M. Armand, B. Pasquier, M. Andre, P. Borel, M. Senft, J. Peyrot, J. Salducci, H. Portugal, V. Jaussan and D. Lairon, Am. J. Clin. Nutr., 1999, 70, 1096-1106.

10 M. Hamosh, J. Bitman, T. H. Liao, N. R. Mehta, R. J. Buczek, D. L. Wood, L. J. Grylack and P. Hamosh, Pediatrics, 1989, 83, 86-92.

11 C. Roman, F. Carriere, P. Villeneuve, M. Pina, V. Millet, U. Simeoni and J. Sarles, Pediatr. Res., 2007, 61, 83-88.

12 S. Bernback, L. Blackberg and O. Hernell, Biochim. Biophys. Acta, 1989, 1001, 286-293.

13 S. Bernback, L. Blackberg and O. Hernell, J. Clin. Invest., 1990, 85, 1221-1226.

14 M. Hamosh, Adv. Exp. Med. Biol., 2002, 503, 17-25.

15 G. Fave, T. C. Coste and M. Armand, Cell. Mol. Biol., 2004, 50, 815-831.

16 N. H. Zangenberg, A. Mullertz, H. G. Kristensen and L. Hovgaard, Eur. J. Pharm. Sci., 2001, 14, 237-244.

17 N. H. Zangenberg, A. Mullertz, H. G. Kristensen and L. Hovgaard, Eur. J. Pharm. Sci., 2001, 14, 115-122.

18 J. C. Bakala-N'Goma, H. D. Williams, P. J. Sassene, K. Kleberg, M. Calderone, V. Jannin, A. Igonin, A. Partheil, D. Marchaud, E. Jule, J. Vertommen, M. Maio, R. Blundell, H. Benameur, A. Mullertz, C. W. Pouton, C. J. Porter and F. Carriere, Pharm. Res., 2015, 32, 1279-1287.

19 P. Sassene, K. Kleberg, H. D. Williams, J. C. BakalaN'Goma, F. Carriere, M. Calderone, V. Jannin, A. Igonin, A. Partheil, D. Marchaud, E. Jule, J. Vertommen, M. Maio, R. Blundell, H. Benameur, C. J. Porter, C. W. Pouton and A. Mullertz, AAPS J., 2014, 16, 1344-1357.
20 H. D. Williams, M. U. Anby, P. Sassene, K. Kleberg, J. C. Bakala-N'Goma, M. Calderone, V. Jannin, A. Igonin, A. Partheil, D. Marchaud, E. Jule, J. Vertommen, M. Maio, R. Blundell, H. Benameur, F. Carriere, A. Mullertz, C. W. Pouton and C. J. Porter, Mol. Pharm., 2012, 9, 3286-3300.

21 H. D. Williams, P. Sassene, K. Kleberg, J. C. BakalaN'Goma, M. Calderone, V. Jannin, A. Igonin, A. Partheil, D. Marchaud, E. Jule, J. Vertommen, M. Maio, R. Blundell, H. Benameur, F. Carriere, A. Mullertz, C. J. Porter and C. W. Pouton, J. Pharm. Sci., 2012, 101, 3360-3380.

22 H. D. Williams, P. Sassene, K. Kleberg, M. Calderone, A. Igonin, E. Jule, J. Vertommen, R. Blundell, H. Benameur, A. Mullertz, C. J. Porter, C. W. Pouton and L. C. Communicated on Behalf of the, J. Pharm. Sci., 2014, 103, 2441-2455.

23 H. D. Williams, P. Sassene, K. Kleberg, M. Calderone, A. Igonin, E. Jule, J. Vertommen, R. Blundell, H. Benameur, A. Mullertz, C. W. Pouton, C. J. Porter and L. Consortium, Pharm. Res., 2013, 30, 3059-3076.

24 A. Dahan and A. Hoffman, Pharm. Res., 2006, 23, 21652174.

25 A. Dahan and A. Hoffman, Eur. J. Pharm. Biopharm., 2007, 67, 96-105.

26 M. Minekus, M. Alminger, P. Alvito, S. Ballance, T. Bohn, C. Bourlieu, F. Carriere, R. Boutrou, M. Corredig, D. Dupont, C. Dufour, L. Egger, M. Golding, S. Karakaya, B. Kirkhus, S. Le Feunteun, U. Lesmes, A. Macierzanka, A. Mackie, S. Marze, D. J. McClements, O. Menard, I. Recio, C. N. Santos, R. P. Singh, G. E. Vegarud, M. S. Wickham, W. Weitschies and A. Brodkorb, Food Funct., 2014, 5, 11131124.

27 R. Barker, B. Abrahamsson and M. Kruusmagi, J. Pharm. Sci., 2014, 103, 3704-3712.

28 P. A. Dickinson, R. Abu Rmaileh, L. Ashworth, R. A. Barker, W. M. Burke, C. M. Patterson, N. Stainforth and M. Yasin, AAPS J., 2012, 14, 196-205.

29 C. J. H. Porter, N. L. Trevaskis and W. N. Charman, Nat. Rev. Drug Discovery, 2007, 6, 231-248.

30 A. T. Larsen, P. Sassene and A. Mullertz, Int. J. Pharm., 2011, 417, 245-255.

31 S. Nury, G. Pieroni, C. Riviere, Y. Gargouri, A. Bois and R. Verger, Chem. Phys. Lipids, 1987, 45, 27-37.

32 Y. Gargouri, G. Pieroni, C. Riviere, J. F. Sauniere, P. A. Lowe, L. Sarda and R. Verger, Gastroenterology, 1986, 91, 919-925.

33 E. Ville, F. Carriere, C. Renou and R. Laugier, Digestion, 2002, 65, 73-81.

34 J. Vecht, T. Symersky, C. B. Lamers and A. A. Masclee, J. Clin. Gastroenterol., 2006, 40, 721-725.

35 E. Rogalska, S. Ransac and R. Verger, J. Biol. Chem., 1990, 265, 20271-20276.

36 L. Sams, J. Paume, J. Giallo and F. Carriere, Food Funct., 2016, 7, 30-45.

37 P. C. Christophersen, M. L. Christiansen, R. Holm, J. Kristensen, J. Jacobsen, B. Abrahamsson and A. Mullertz, Eur. J. Pharm. Sci., 2014, 57, 232-239. 
38 E. Rogalska, C. Cudrey, F. Ferrato and R. Verger, Chirality, 1993, 5, 24-30.

39 A. Mercuri, A. Lo Curto, M. S. J. Wickham, D. Q. M. Craig and S. A. Barker, J. Pharm. Pharmacol., 2008, 60, A2-A2.

40 A. Mercuri, A. Passalacqua, M. S. J. Wickham, R. M. Faulks, D. Q. M. Craig and S. A. Barker, Pharm. Res., 2011, 28, 1540-1551.

41 M. J. S. Wickham, R. M. Faulks, J. Mann and G. Mandalari, Dissolution Technol., 2012, 19, 15-22.

42 M. Minekus, P. Marteau, R. Havenaar and J. H. J. Huisintveld, ATLA, Altern. Lab. Anim., 1995, 23, 197-209.

43 L. Sek, C. J. H. Porter, A. M. Kaukonen and W. N. Charman, J. Pharm. Pharmacol., 2002, 54, 29-41.

44 J. O. Christensen, K. Schultz, B. Mollgaard, H. G. Kristensen and A. Mullertz, Eur. J. Pharm. Sci., 2004, 23, 287-296.

45 F. Carriere, H. Moreau, V. Raphel, R. Laugier, C. Benicourt, J. L. Junien and R. Verger, Eur. J. Biochem./FEBS, 1991, 202, 75-83.

46 H. Moreau, Y. Gargouri, D. Lecat, J. L. Junien and R. Verger, Biochim. Biophys. Acta, 1988, 960, 286-293.

47 P. Capolino, C. Guérin, J. Paume, J. Giallo, J. Ballester, J. Cavalier and F. Carrière, Food Dig., 2011, 2, 43-51.

48 S. Fernandez, S. Chevrier, N. Ritter, B. Mahler, F. Demarne, F. Carriere and V. Jannin, Pharm. Res., 2009, 26, 19011910.

49 Y. Yang and D. J. McClements, Food Chem., 2013, 141, 473481.

50 C. J. Porter, A. M. Kaukonen, B. J. Boyd, G. A. Edwards and W. N. Charman, Pharm. Res., 2004, 21, 1405-1412.

51 S. Canaan, L. Dupuis, M. Riviere, K. Faessel, J. L. Romette, R. Verger and C. Wicker-Planquart, Protein Expression Purif., 1998, 14, 23-30.

52 T. Crabbe, A. N. Weir, E. F. Walton, M. E. Brown, C. W. Sutton, N. Tretout, J. Bonnerjea, P. A. Lowe and G. T. Yarranton, Protein Expression Purif., 1996, 7, 229-236.

53 P. Sassene, M. Fanø, P. Ravn, T. T. T. N. Nguyen, Z. E. Nazari, K. D. Rand, H. Mu, T. Rades and A. Mullertz, in preparation, 2016.
54 M. Hamosh, J. W. Scanlon, D. Ganot, M. Likel, K. B. Scanlon and P. Hamosh, J. Clin. Invest., 1981, 67, 838846.

55 D. N. Challacombe, S. Edkins and G. A. Brown, Arch. Dis. Child., 1975, 50, 837-843.

56 J. F. T. Glasgow, H. Dinsmore, A. Molla and T. Macfarlane, Ir. J. Med. Sci., 1980, 149, 346-356.

57 J. B. Watkins, P. Szczepanik, J. B. Gould, P. Klein and R. Lester, Gastroenterology, 1975, 69, 706-713.

58 A. L. Jarvenpaa, D. K. Rassin, P. Kuitunen, G. E. Gaull and N. C. R. Raiha, Pediatrics, 1983, 72, 677-683.

59 B. L. Dekkers, E. Kolodziejczyk, S. Acquistapace, J. Engmann and T. J. Wooster, Food Funct., 2016, 7, $58-68$.

60 L. Kalantzi, K. Goumas, V. Kalioras, B. Abrahamsson, J. B. Dressman and C. Reppas, Pharm. Res., 2006, 23, 165176.

61 F. Destaillats and C. Cruz-Hernandez, J. Chromatogr., A, 2007, 1169, 175-178.

62 C. Cruz-Hernandez, Z. Y. Deng, J. Q. Zhou, A. R. Hill, M. P. Yurawecz, P. Delmonte, M. M. Mossoba, M. E. R. Dugan and J. K. G. Kramer, J. AOAC Int., 2004, 87, 545-562.

63 Y. Pafumi, D. Lairon, P. L. de la Porte, C. Juhel, J. Storch, M. Hamosh and M. Armand, J. Biol. Chem., 2002, 277, 28070-28079.

64 E. M. Carey and R. Dils, Biochem. J., 1972, 126, 1005-1007.

65 S. Gallier, J. Cui, T. D. Olson, S. M. Rutherfurd, A. Q. Ye, P. J. Moughan and H. Singh, Food Chem., 2013, 141, 32733281.

66 F. Carriere, P. Grandval, C. Renou, A. Palomba, F. Prieri, J. Giallo, F. Henniges, S. Sander-Struckmeier and R. Laugier, Clin. Gastroenterol. Hepatol., 2005, 3, 28-38.

67 L. D. Mendoza, J. A. Rodriguez, J. Leclaire, G. Buono, F. Fotiadu, F. Carrière and A. Abousalham, J. Lipid Res., 2012, 53, 185-194.

68 S. Fernandez, V. Jannin, J. D. Rodier, N. Ritter, B. Mahler and F. Carriere, Biochim. Biophys. Acta, 2007, 1771, 633-640. 